\title{
Omissão de fármacos modificadores do risco cardiovascular em idosos admitidos numa Unidade de Acidentes Vasculares Cerebrais
}

Elisabete Pinto Borges*, Ana Filipa Macedo**

\section{RESUMO}

Objectivos: Quantificar e caracterizar omissões de prescrição de fármacos modificadores do risco cardiovascular utilizando os critérios START (Screening Tool to Alert Doctors to The Right Treatment).

Tipo de Estudo: Estudo observacional descritivo.

Local: Unidade de Acidentes Vasculares Cerebrais (UAVC) do Centro Hospitalar da Cova da Beira (CHCB).

População: Idosos (idade $\geq 65$ anos) internados com doença aguda cardiovascular.

Métodos: Os processos clínicos de todos os idosos internados entre 1 de Outubro e 31 de Dezembro de 2009 foram revistos e os critérios START aplicados à medicação nos momentos de admissão e de alta clínica.

Resultados: Durante o período de estudo foram admitidos na UAVC 56 doentes idosos. No momento de internamento detectaram-se 63 omissões de prescrição em $69,8 \%$ dos idosos (média 1,19 omissões por doente), que em $74,5 \%$ ( $n=38$ ) dos casos foram corrigidas no momento de alta. Nos doentes medicados com 5 ou mais medicamentos diários detectaram-se omissões de prescrição à admissão em $80,9 \%$ dos casos. Em 10 doentes, 13 omissões detectadas à entrada permaneciam por corrigir e em 3 doentes registaram-se 3 novas omissões de prescrição.

Os critérios START identificaram, tanto na entrada hospitalar como na alta clínica, omissões de prescrição sobretudo de medicação do sistema cardiovascular e do sistema endócrino.

Conclusões: A omissão de fármacos em idosos admitidos no hospital com doença aguda cardiovascular é frequente. Os critérios START revelaram-se um instrumento útil de validação da prescrição farmacológica, de acordo com a evidência das normas de orientação clínica actuais, sobretudo em termos de prevenção cardiovascular.

Palavras-Chave: Idoso; Reacção Adversa a Medicamento; Prescrição de Medicamentos; Acidente Vascular Cerebral; Doenças Cardiovasculares.

\section{INTRODUÇÃO}

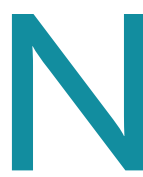

os países industrializados uma parte significativa da população tem mais de 65 anos, e esta parcela tende a aumentar. $\mathrm{O}$ envelhecimento da população constitui

*Mestre em Medicina pela Faculdade de Ciências da Saúde da Universidade da Beira Interior.

**Doutora em Farmácia na Especialidade de Farmácia Clínica. Professora Auxiliar da Faculdade de Ciências da Saúde da Universidade da Beira Interior. Membro do Centro de Investigação em Ciência da Saúde (CICS) da Universidade da Beira Interior, Covilhã. Membro do Conselho Cientifico da Unidade de Farmacovigilância do Centro, AIBILI, Coimbra. um dos aspectos mais marcantes da evolução demográfica recente, com fortes implicações estruturantes, sobretudo no sector da saúde, traduzidas num maior consumo de medicamentos e numa procura cada vez maior de cuidados de especialidade e cuidados continuados. ${ }^{1}$

Com o envelhecimento populacional aumenta a prevalência de doenças crónicas e incapacitantes, como as doenças cardiovasculares (DCV), o cancro, a diabetes e as doenças respiratórias, responsáveis actualmente por $60 \%$ das mortes a nível mundial. ${ }^{2}$ Na população por- 
tuguesa, as DCV constituem a primeira causa de morte prematura e uma importante causa de incapacidade, fundamentalmente como consequência do acidente vascular cerebral (AVC) e da doença coronária, com maior expressão a partir dos 65 anos (42\% do total de mortes). ${ }^{2,3}$

Este aumento da prevalência de doenças crónicas na população idosa conduz inevitavelmente a um aumento do consumo de medicamentos, particularmente fármacos modificadores do risco cardiovascular, anti-inflamatórios não esteróides (AINE's) e medicamentos psicotrópicos. ${ }^{4,5} \mathrm{~A}$ polimedicação, juntamente com alterações farmacocinéticas e farmacodinâmicas próprias da idade e, por vezes, alterações cognitivas que comprometem o cumprimento do regime terapêutico, ${ }^{6}$ predispõem a população idosa a problemas relacionados com medicamentos (PRM). ${ }^{7,8}$ Os PRM abrangem o uso excessivo ou inapropriado de fármacos, a utilização de fármacos fracamente tolerados ou a subutilização de medicação considerada necessária, com probabilidade de exacerbar uma condição clínica. ${ }^{7,9,10}$

Vários estudos sugerem que os PRM no idoso são responsáveis por 3 a 12\% de admissões hospitalares e mais de $50 \%$ podem ser prevenidos, sobretudo causados por prescrição inapropriada (PI). ${ }^{11,12,13}$ Este é amplamente reconhecido como um problema de saúde pública, o que torna a optimização da prescrição para este grupo de doentes uma componente fundamental da farmacoterapia geriátrica. A detecção precoce de PRM, identificando oportunidades de prevenção e diminuição das suas consequências, é hoje imperativa em cuidados geriátricos. ${ }^{14}$

Vários critérios têm sido publicados para auxiliar os clínicos a identificar PRM devido a PI. ${ }^{3-20}$ No entanto, no que se refere a critérios que auxiliem a identificação da subutilização de medicação considerada benéfica, as publicações são escassas, sendo este um problema com significativa prevalência entre idosos que recorrem ao hospital com doença aguda. ${ }^{10}$ Recentemente foram publicados os critérios START «Screening Tool to Alert Doctors to the Right Treatment», que constituem um método de identificação sistemática de omissão de prescrições, baseado em sistemas fisiológicos..$^{20,21}$ Os critérios START foram validados através do processo de Delphi, por 18 especialistas em geriatria, apresentando um grau de concordância razoável entre avaliadores (coeficien- te Kappa 0,68). Após a sua validação, os critérios START foram aplicados às informações de diagnóstico e de prescrição farmacológica de uma amostra prospectiva, aleatória consecutiva, de 600 idosos (idade $\geq 65$ anos) provenientes da comunidade e admitidos por doença aguda num hospital universitário, tendo-se identificado uma ou mais omissões de prescrição em 57,9\% dos doentes. ${ }^{21}$ Neste estudo os critérios START foram considerados válidos, efectivos e fáceis de usar na identificação de omissão inapropriada de terapêutica essencial. $^{21}$

Atendendo à elevada prevalência e mortalidade por DCV da população idosa portuguesa, torna-se fundamental assegurar neste grupo populacional uma prescrição preventiva adequada à redução do risco global, a longo prazo, de doença e morte cardiovascular. ${ }^{22} \mathrm{O}$ presente estudo pretendeu, por isso, quantificar e caracterizar problemas de omissão de prescrição de fármacos modificadores do risco cardiovascular na população idosa internada com doença aguda do foro cardiovascular, detectados mediante utilização dos critérios START.

\section{MÉTODOS}

Efectuou-se um estudo epidemiológico descritivo, incluindo todos os doentes idosos (idade $\geq 65$ anos) internados na Unidade de Acidentes Vasculares Cerebrais (UAVC) do Centro Hospitalar da Cova da Beira (CHCB) entre 1 de Outubro e 31 de Dezembro de 2009. Nas UAVC são admitidos doentes com início súbito de défices neurológicos focais de presumível origem vascular, com a finalidade de iniciar precocemente o tratamento e a neuro-reabilitação, prevenir complicações e recorrências, identificar factores de risco, tratar comorbilidades e desenvolver um plano de alta e de monitorização adequados.

Após a aprovação do protocolo do estudo pelo conselho de administração do CHCB, um investigador independente consultou os processos clínicos, recolhendo informação sobre antecedentes clínicos, informação sobre a medicação em ambulatório e o motivo de internamento - referidos na nota de entrada-bem como a medicação prescrita e diagnósticos de alta - referidos na nota de alta. Adicionalmente, foi obtida informação demográfica de cada doente (idade e género) e informação de exames complementares de diagnóstico efec- 
tuados. Os dados recolhidos foram analisados utilizando os critérios START para identificar omissões de prescrição no momento de internamento («entrada») e no momento da alta clínica («saída»).

Os critérios START incorporam 22 indicadores de potenciais omissões de prescrição em idosos, agrupados em sistemas fisiológicos. No presente estudo apenas foram utilizados os indicadores do sistema cardiovascular e do sistema endócrino (Quadro I), que sinalizam omissão de prescrição fármacos modificadores do risco cardiovascular.

De notar que a tradução adoptada não foi validada pelo método «traduz - retraduz», pelo que qualquer dúvida de linguagem ou gramática deve ser esclarecida com consulta da versão original. ${ }^{20}$

Para codificar as omissões de prescrição foi atribuída a letra A ao sistema cardiovascular e foi atribuída a letra $\mathrm{F}$ ao sistema endócrino. A cada critério foi atribuído um número (ex. A8 - Critério 8 do Sistema Cardiovascular). Em relação ao critério A4, não foi considerado na análise o limite de $160 \mathrm{mmHg}$, proposto pelos critérios START, por falta de registos dos valores de tensão arterial nos processos clínicos, considerando-se apenas a referência ou não a antecedentes de hipertensão arterial. No critério A5 não foi considerado o limite de 5 anos para a Esperança Média de Vida (EMV) pois na literatura recente a terapêutica com estatinas mostra ainda benefícios em idades superiores, pelo que foi considerada a idade limite de 85 anos. ${ }^{23,24}$ Sempre que se observou qualquer contra-indicação a uma terapêutica sinalizada nos critérios START, a referida situação não foi considerada omissão de prescrição.

Os dados recolhidos foram tratados no programa SPSS (Statistical Package for the Social Sciences) versão 17.0.

\section{RESULTADOS}

Durante o período de estudo foram admitidos na UAVC 56 doentes idosos, maioritariamente do sexo feminino (51,8\%), cujas idades variaram entre os 65 e os 98 anos (média 78,05 $\pm 6,87$ anos). Mais de 50\% dos doentes pertenciam à faixa etária dos 70-80 anos.

O principal motivo de admissão hospitalar foi o AVC isquémico (71,4\%), seguido do AVC hemorrágico e AIT (14,3\% cada).

Devido às particularidades dos doentes admitidos

\section{QUADRO I. Critérios START ${ }^{20}$}

Sistema Cardiovascular

A1 - Varfarina na presença de fibrilhação auricular

A2 - Aspirina na presença de fibrilhação auricular crónica, quando a varfarina é contra-indicada, mas não a aspirina

A3 - Aspirina ou clopidogrel com história documentada de aterosclerose coronária, cerebral ou doença vascular periférica em idosos com ritmo sinusal

A4 - Terapêutica anti-hipertensiva quando pressão sanguínea sistólica persistente $>160 \mathrm{mmHg}$

A5 - Terapêutica com estatinas com história documentada de doença coronária, cerebral, ou vascular periférica, quando o estado funcional do idoso permite independência nas actividades de vida diárias e a esperança média de vida é maior do que 5 anos

A6 - Inibidores da enzima de conversão da angiotensina (IECA's) na insuficiência cardíaca crónica

A7 - IECA's após enfarte agudo do miocárdio

A8 - $\beta$-Bloqueantes na angina crónica estável

Sistema Endócrino

F1 - Metformina em diabéticos tipo 2 +/- Síndrome metabólico (na ausência de insuficiência renal)

F2 - Inibidores ou bloqueadores do receptor da enzima de conversão da angiotensina em diabetes com nefropatia, ex. análise urinária com proteinúria ou microalbuminúria (>30 $\mathrm{mg} / 24$ horas) +/- bioquímica da função renal alterada

F3 - Terapêutica com antiagregante plaquetário em diabetes mellitus com factores de risco cardiovascular major coexistentes (hipertensão, hipercolesterolémia, história de tabagismo)

F4 - Terapêutica com estatinas em diabetes mellitus se factores de risco cardiovascular major coexistentes

na UAVC, os antecedentes clínicos eram sobretudo cardiovasculares $(91,1 \%)$ e endócrinos $(34,0 \%)$ (Quadro II). A medicação de ambulatório incluía sobretudo fármacos com acção no sistema cardiovascular $(80,4 \%)$, no sistema nervoso central $(46,4 \%)$ e no sistema endócrino $(32,2 \%)$. Os idosos incluídos no estudo tomavam em média 4,62 medicamentos por doente (mínimo 0; máximo 12) e 37,5\% ( $n=21)$ estavam medicados com 5 ou mais medicamentos, simultaneamente (polimedicados). 
QUADRO II. Antecedentes clínicos e medicação em ambulatório, por grupo fisiológico.

\begin{tabular}{|c|c|c|}
\hline & $\begin{array}{l}\text { Antecedentes Clínicos } \\
\text { (\% de idosos) [IC 95\%] }\end{array}$ & $\begin{array}{l}\text { Medicação de Ambulatório } \\
\text { (\% de idosos) [IC 95\%] }\end{array}$ \\
\hline Sistema Cardiovascular & $91,1[83,5-98,5]$ & $80,4[69,95-90,77]$ \\
\hline Sistema Respiratório & $8,9[1,46-16,4]$ & $3,6[-1,29-8,43]$ \\
\hline Sistema Nervoso Central & $25,0[13,66-36,4]$ & $46,4[33,37-59,49]$ \\
\hline Sistema Gastrointestinal & $8,9[1,46-16,4]$ & $21,5[10,68-32,18]$ \\
\hline Sistema Músculo-esquelético & $21,5[10,68-32,18]$ & $26,8[15,19-38,39]$ \\
\hline Sistema Endócrino & $34,0[21,53-46,33]$ & $32,2[19,91-44,37]$ \\
\hline Outras patologias & $30,5[18,32-42,4]$ & $16,1[6,45-25,69]$ \\
\hline $\begin{array}{l}\text { Antecedentes/Medicação } \\
\text { desconhecidos }\end{array}$ & $3,6[-1,29-8,43]$ & $1,8[-1,68-5,26]$ \\
\hline
\end{tabular}

um total de 16 omissões de prescrição, em média 0,36 omissões por doente, registando-se um máximo de 2 omissões num mesmo doente.

Os critérios START permitiram identificar omissões de prescrição de medicação do sistema cardiovascular em $62,3 \%(n=33)$ dos idosos na entrada hospitalar e em $27,3 \%(n=12)$ idosos na alta clínica; e omissões do sistema endócrino em $22,6 \%(n=12)$ idosos na en-

Em 3 dos 56 idosos não foi possível aplicar os critérios START no momento da entrada na UAVC, por se desconhecerem os antecedentes clínicos em dois desses doentes e noutro por se desconhecer quais os medicamentos em ambulatório. No momento de alta clínica, foi possível aplicar os critérios START a todos os doentes.

A aplicação dos critérios START ao regime terapêutico do momento de internamento (correspondente à medicação de ambulatório) permitiu identificar omissões de prescrição em 69,8\% (n=37) dos 53 doentes (Quadro III). Foi registado um total de 63 omissões de prescrição, em média 1,19 omissões por doente, observando-se um máximo de 3 omissões num mesmo doente.

Dos doentes medicados simultaneamente com 5 ou mais medicamentos, 80,9\% (n=17) apresentaram omissões de prescrição.

À saída da UAVC a amostra ficou reduzida a 44, uma vez que 12 doentes faleceram. A aplicação dos critérios START ao regime terapêutico do momento da alta clínica permitiu identificar omissões de prescrição em 27,3\% (n=12) dos doentes (Quadro III). Foi registado trada e 6,8\% ( $\mathrm{n}=3$ ) idosos na alta (Quadro III).

No momento de internamento, a principal omissão de prescrição do sistema cardiovascular foi a da terapêutica com estatinas em doentes com história documentada de doença coronária, cerebral, ou vascular periférica (A5), representando $27,9 \%(\mathrm{n}=12)$ do total de omissões do grupo A ( $\mathrm{n}=43$ ) (Figura 1). Seguiram-se as omissões

\begin{tabular}{|c|c|c|}
\hline \multicolumn{3}{|c|}{$\begin{array}{l}\text { QUADRO III. Doentes com omissões de prescrição (OP) identificadas pelos } \\
\text { critérios START à entrada e à saída da UAVC }\end{array}$} \\
\hline & \multicolumn{2}{|c|}{ Unidade de AVC } \\
\hline & Entrada & Saída \\
\hline Total de OP (N) & 63 & 16 \\
\hline $\begin{array}{l}\text { Doentes com OP } \\
\qquad N(\%)[I C 95 \%]\end{array}$ & $37^{*}(69,8)[57,45-82,17]$ & $12^{* *}(27,3)[14,11-40,43]$ \\
\hline Média OP/Doente [IC95\%] & $1,19[0,91-1,46]$ & $0,36[0,17-0,56]$ \\
\hline \multicolumn{3}{|l|}{$\begin{array}{l}\text { OP simultâneas } \\
\qquad \mathrm{N}(\%) \text { [IC95\%] }\end{array}$} \\
\hline 1 & $17(32,1)[19,51-44,65]$ & $8(18,2)[6,78-29,58]$ \\
\hline 2 & $14(26,4)[14,55-38,29]$ & $4(9,1)[0,6-17,58]$ \\
\hline$\geq 3$ & $6(11,3)[2,79-19,85]$ & 0,0 \\
\hline \multicolumn{3}{|l|}{$\begin{array}{l}\text { Doentes com OP } \\
\text { N (\%) [IC95\%] }\end{array}$} \\
\hline Cardiovascular & $33(62,3)[49,21-75,31]$ & $12(27,3)[14,11-40,43]$ \\
\hline Endócrino & $12(22,6)[11,37-33,91]$ & $3(6,8)[-0,63-14,27]$ \\
\hline
\end{tabular}

*Em 3 dos 56 idosos não foi possível aplicar os critérios START no momento da entrada na UAVC **À saída da UAVC a amostra ficou reduzida a 44, uma vez que 12 doentes faleceram 
de terapêutica anti-hipertensiva (A4) e de varfarina na presença de fibrilhação auricular (A1), ambas com uma proporção de $18,6 \%$ $(\mathrm{n}=8)$.

$\mathrm{Na}$ alta clínica, as únicas omissões de prescrição do sistema cardiovascular registadas foram a terapêutica com estatinas em idosos com história documentada de doença coronária, cerebral, ou vascular periférica (A5) e a prescrição de $\beta$-bloqueantes na angina crónica estável (A8), correspon-

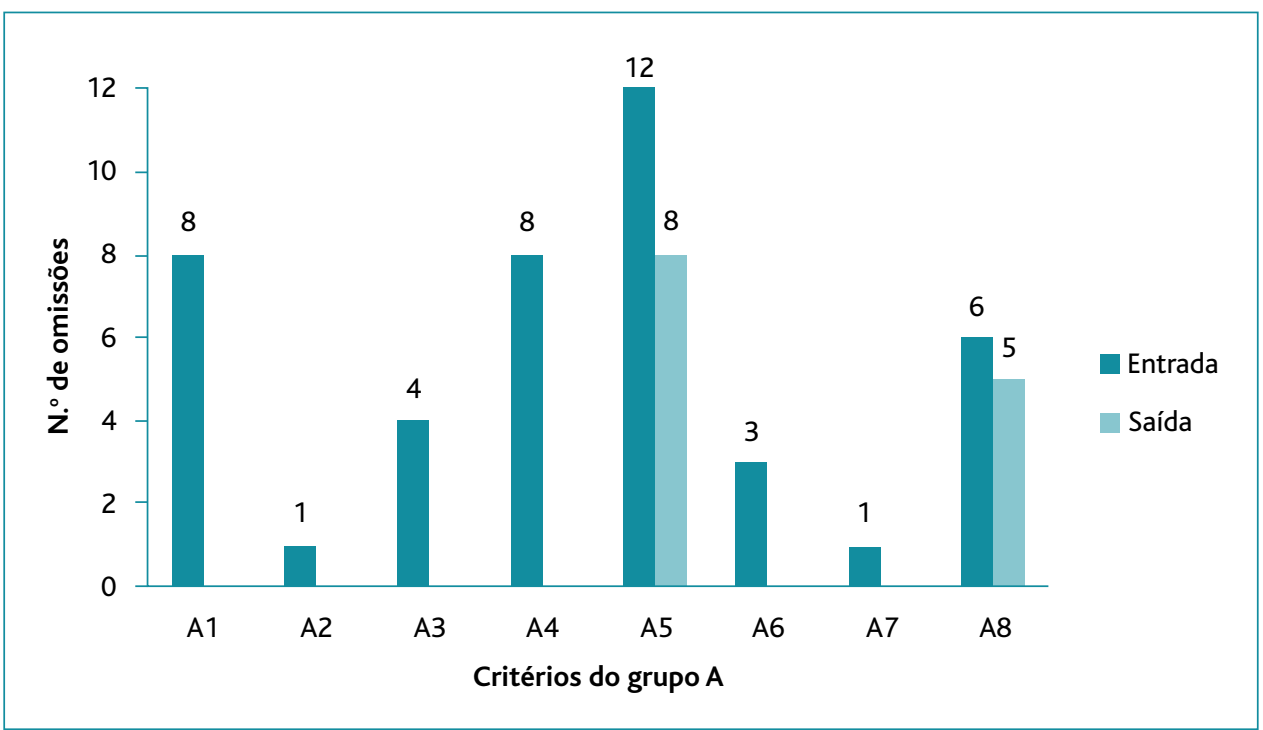

Figura 1. Omissões de prescrição do grupo A (sistema cardiovascular). dendo a $61,5 \%(\mathrm{n}=8)$ e $38,5 \%(n=5)$ do total de omissões do grupo $A(n=13)$, respectivamente.

Relativamente ao sistema endócrino (Figura 2), o principal critério omitido à entrada foi a terapêutica com estatinas em doentes com diabetes mellitus e factores de risco cardiovascular major coexistentes (F4), correspondendo a $45,0 \%(n=9)$ do total de omissões do grupo $F(n=20)$. Seguiu-se a omissão de terapêutica antiagregante plaquetária em diabéticos com factores de risco cardiovascular major coexistentes (F3), representando $40,0 \%(\mathrm{n}=8)$ das omissões. À saída $6,8 \%(n=3)$ dos doentes apresentavam omissões sinalizadas pelo critério F4.

As omissões de prescrição identificadas à entrada na UAVC foram corrigidas em 74,5\% ( $\mathrm{n}=38)$ dos casos (Figura 3). No momento da alta clínica, 13 omissões num total de 10 doentes permaneciam por corrigir: em 5 doentes com história documentada de doença coronária, cerebral ou vascular periférica não foi corrigida a falta de terapêutica com estatinas (A5), a omissão da utilização de $\beta$-bloqueantes não foi corrigida em 5 doentes com angina crónica estável (A8) e 3 diabéticos com factores de risco cardiovascular major coexistentes tiveram alta clínica sem prescrição de estatinas (F4).

Em 3 idosos foram identificadas 3 novas omissões no momento da alta clínica, correspondentes à falta de prescrição de estatinas após história documentada de doença coronária, cerebral ou vascular periférica (A5).

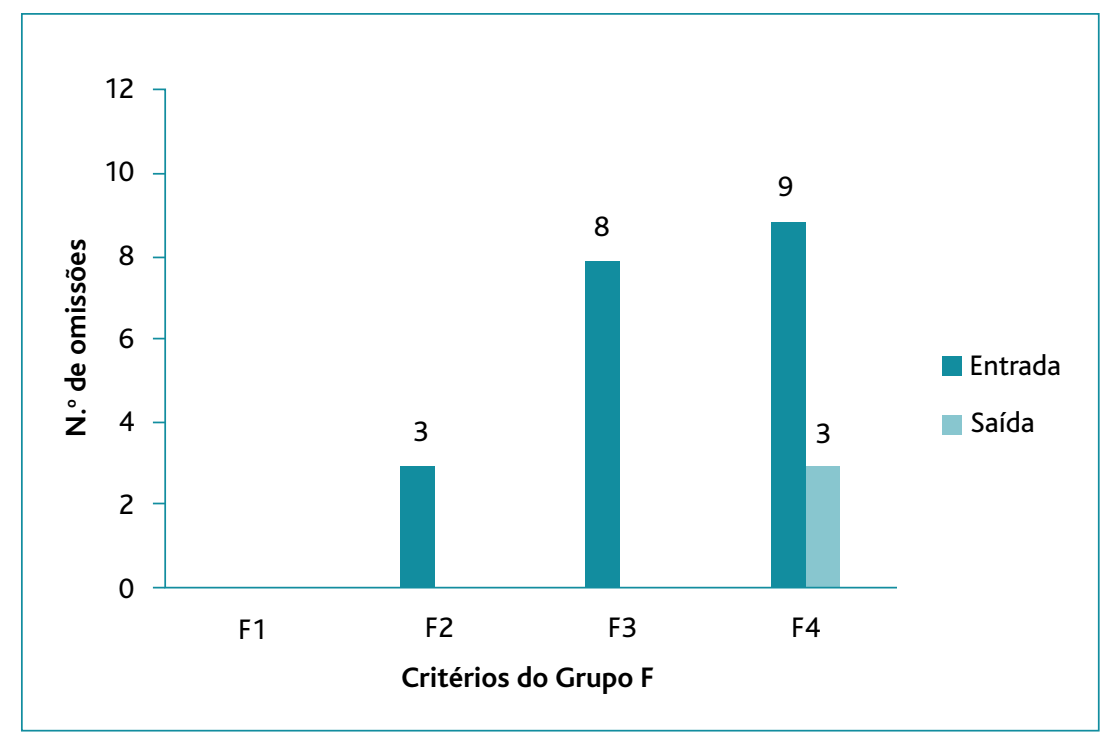

Figura 2. Omissões de prescrição do Grupo F (sistema endócrino). 


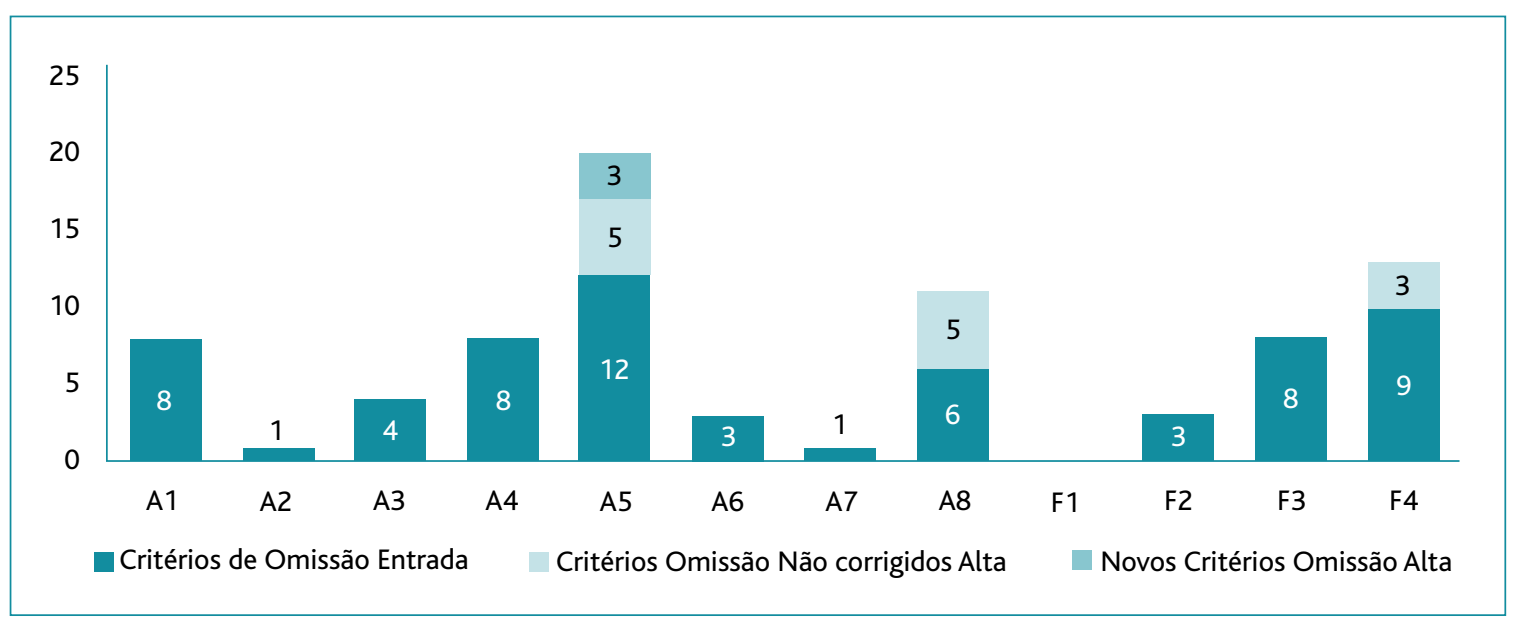

Figura 3. Correcção das omissões de prescrição à entrada na alta clínica.

\section{DISCUSSÃO}

Os problemas relacionados com a medicação (PRMs) são uma importante causa de morbilidade e mortalidade da população idosa, na maioria dos casos evitável. Existem diversas publicações sobre indicadores de prescrição medicamentosa que permitem a identificação de prescrições inapropriadas que são causas de PRMs. ${ }^{11,12,13}$ Contudo, não existem dados epidemiológicos actuais sobre indicadores que permitam identificar omissão de medicação considerada benéfica na população idosa.

No presente estudo, a utilização dos critérios START permitiu a identificação sistemática de omissões de medicação em $69,8 \%$ dos idosos, no momento de internamento numa UAVC. As omissões de prescrição identificadas à entrada na UAVC foram corrigidas durante o internamento hospitalar em $74,5 \%(n=38)$ dos casos. No entanto, no momento da alta clínica, a aplicação dos critérios START ao regime terapêutico permitiu ainda identificar 16 omissões de prescrição em $29,3 \%(n=12)$ dos doentes. Destas, 13 eram omissões detectadas à entrada em 10 doentes e que permaneciam por corrigir, e 3 novas omissões em 3 doentes, uma delas referente a um doente a quem à entrada não foi possível aplicar os critérios START.

Na nota de entrada do doente consta apenas a medicação em ambulatório no momento de admissão no hospital, pelo que não foi possível esclarecer as causas das omissões de medicação detectadas. Do mesmo modo, as notas de alta clínica não apresentavam qual- quer registo a justificar as omissões de medicação detectadas. A não utilização de um medicamento considerado necessário pode dever-se à não prescrição por parte dos clínicos (por omissão ou justificada por princípios da proporcionalidade e da precaução iatrogénica - benefícios esperados devem superar os riscos eventuais), ou a situações como a não adesão à terapêutica por parte do idoso, ou a não aquisição de um medicamento de custo elevado, que fazem com que o doente não use determinado medicamento, apesar de prescrito. De qualquer modo, estes resultados demonstram que a subutilização de medicação considerada necessária é uma realidade não negligenciável entre os doentes idosos admitidos com doença aguda.

Em 80,9\% dos doentes medicados com 5 ou mais medicamentos diários foram detectadas omissões de prescrição na admissão, evidenciando que mesmo os doentes polimedicados são frequentemente subtratados. Estudos epidemiológicos semelhantes observaram também elevada prevalência de subutilização de medicação considerada necessária e de medicação inapropriada em doentes polimedicados..$^{25,26} \mathrm{~A}$ polimedicação é frequentemente conotada como prejudicial por estar associada a um aumento do risco de efeitos adversos. No entanto, a presença de co-morbilidades na população idosa e a afirmação crescente da medicina preventiva aumentam cada vez mais a racionalidade de uma prescrição múltipla. ${ }^{27} \mathrm{~A}$ detecção dos fármacos omitidos nos idosos polimedicados pode, por isso, tornar-se uma tarefa de considerável complexidade, pelo 
que a utilização dos critérios START se revela uma ferramenta auxiliar importante neste contexto.

A principal omissão do sistema cardiovascular à entrada da UAVC foi a terapêutica com estatinas em doentes com história documentada de doença coronária, cerebral, ou vascular periférica (A5 - 27,9\% do total de omissões do grupo A). Esta, juntamente com a omissão de prescrição de $\beta$-bloqueantes na angina crónica estável (A8), constituíram as duas únicas omissões do grupo A no momento da alta clínica (A5 e A8 - 61,5\% e $38,5 \%$ do total de omissões do grupo A, respectivamente). O critério A5 foi também o único em que se registaram novas omissões na alta clínica (em 3 idosos).

As estatinas diminuem significativamente a incidência de AVC e EAM, estando, por isso, indicadas quando há história de doença arteriosclerótica. ${ }^{28-30}$ Apesar disso, pela falta de estudos em doentes idosos, a sua utilização neste grupo etário é frequentemente questionada. Porém, evidência científica recente revela um benefício da utilização de estatinas em doentes idosos ao nível da prevenção primária e secundária, igual ou superior ao dos doentes mais jovens. O aumento do risco de acontecimentos adversos (mialgia e elevação das enzimas hepáticas), sobretudo nos doentes mais idosos, requer, no entanto, uma avaliação cuidada das comorbilidades, qualidade de vida e esperança média de vida, no momento de prescrição. ${ }^{23,24,31}$

Além disso, os critérios START devem ser aplicados de acordo com as orientações da ATPIII (The National Cholesterol Education Program Adult Treatment Panel III), que recomendam, em prevenção secundária, níveis de $\mathrm{LDL}<100 \mathrm{mg} / \mathrm{dL}$ e sempre que possível níveis de HDL $>40 \mathrm{mg} / \mathrm{dL}^{31}$

Em relação ao critério A8, a relutância na prescrição de $\beta$-Bloqueantes em idosos pode estar relacionada com o receio da não tolerância destes fármacos, mas as evidências actuais mostram que grande parte dos efeitos adversos (hipotensão, bradicardia, aumento da resistência à insulina, etc.), são dose-dependentes, não ocorrendo quando se inicia a terapêutica com uma dose baixa e se aumenta gradualmente. ${ }^{32}$ Também a possibilidade de a utilização de $\beta$-bloqueantes agravar a arteriopatia periférica, devido ao bloqueio de receptores $\beta_{2}$ que medeiam a vasodilatação ao nível músculo-esquelético, tem levantado preocupações quanto à segurança da sua utilização nos idosos. No entanto, sempre que a arteriopatia periférica for leve a moderada estes fármacos não agravam a capacidade de deambular ou os sintomas de claudicação intermitente nestes doentes. ${ }^{32}$ Deste modo, os $\beta$-bloqueantes são considerados seguros como medicação de primeira linha no tratamento da angina crónica estável em idosos. . $^{33,34}$

Além da omissão de estatinas e $\beta$-bloqueantes, registaram-se omissões de terapêutica anti-hipertensiva (A4) em doentes mencionados como hipertensos nos processos clínicos, e de varfarina na presença de fibrilhação auricular (A1) (ambas em 18,6\% dos casos), que foram totalmente corrigidas no momento da alta clínica.

É consensual que a hipertensão arterial (HTA) aumenta a incidência de doença coronária, AVC, insuficiência cardíaca, doença arterial periférica e insuficiência renal, bem como o risco de deterioração cognitiva e demência. ${ }^{22}$ Para o seu controlo, são recomendadas alterações do estilo de vida e terapêutica farmacológica adicional quando essas medidas são insuficientes. ${ }^{35,36}$ Relativamente ao critério Al, sabe-se que a fibrilhação auricular é uma importante causa de tromboembolismo, com elevada expressão nos doentes idosos, e que o uso de terapêutica antitrombótica diminui a mortalidade por eventos isquémicos. ${ }^{37,38} \mathrm{Uma}$ das principais razões da omissão de anticoagulantes em idosos é o receio de hemorragias, particularmente nos mais idosos (>75anos). Ainda assim, devido ao elevado risco de AVC nestes doentes, o uso de anticoagulantes pode ter benefícios superiores aos verificados em doentes mais jovens. Como tal, recomenda-se para os doentes com menos de 75 anos, sem contra-indicações para a anticoagulação, um INR de 2,5 (entre 2 e 3) e para aqueles com mais de 75 anos e risco mais acentuado de hemorragia, recomenda-se, como prevenção primária, um INR de 2 (entre 1,6 e 2,5) ${ }^{38}$ Os critérios START podem constituir uma mais-valia ao nível da prevenção secundária, ao alertar os clínicos para esta indicação terapêutica; no entanto não fazem distinção entre idosos com mais e com menos de 75 anos. Além disso não mencionam a utilização do índice CHADS2 (Cardiac Failure, Hypertension, Age, Diabetes, Stroke [Double]) na estimativa do risco de AVC isquémico em doentes idosos com fibrilhação auricular, importante na selecção de fármacos antitrombóticos. ${ }^{38}$

Relativamente ao sistema endócrino, as principais omissões registadas na admissão hospitalar foram as 
estatinas, em idosos diabéticos com factores de risco cardiovascular major coexistentes (F4), e a omissão de terapêutica antiagregante plaquetária, em idosos diabéticos com factores de risco cardiovascular major coexistentes (F3) (F4 e F3 - 45,0\% e 40,0\% do total de omissões do grupo $F$, respectivamente). O critério F4 foi o único em que se detectaram omissões no momento da alta clínica.

A utilização de estatinas é recomendada em todos os idosos diabéticos com DCV prévia, independentemente dos níveis de LDL. Nos idosos diabéticos sem DCV, a terapêutica com estatinas deve ser considerada nos diabéticos tipo 2 com níveis de colesterol total $>3,5$ $\mathrm{mmol} / \mathrm{L}$ (135 mg/dL) e nos diabéticos tipo $1 \mathrm{com}$ mais de 40 anos. ${ }^{39,40,41}$

Os antiagregantes plaquetários são recomendados como prevenção secundária em doentes idosos com evidência de doença aterosclerótica dos grandes vasos. Como prevenção primária, o balanço entre benefício dos antiagregantes e risco de hemorragia não é claro. No entanto, na presença de factores de risco major (hipertensão arterial, tabagismo, hipercolesterolémia) os benefícios superam os riscos devendo-se considerar a utilização destes fármacos. ${ }^{42}$

Em relação aos critérios START, a sua aplicação revelou-se simples e explícita (não requer julgamento clínico), o que diminui o possível enviesamento da avaliação efectuada por uma única pessoa. O tempo normalmente dispendido na aplicação dos critérios START foi mínimo, e por isso compatível com a rotina diária de monitorização farmacoterapêutica.

Para uma optimização da terapêutica, os critérios START deveriam ser combinados com instrumentos de identificação de prescrições farmacológicas inapropriadas. ${ }^{20}$ Além disso, as medidas terapêuticas não farmacológicas, como são as recomendações dietéticas, a cessação tabágica, o exercício físico e o suporte social e familiar, também deveriam ser alvo de avaliação.

Os nossos resultados necessitam de confirmação prospectiva com amostras mais robustas e outras populações de idosos (ambulatório e outros serviços hospitalares). São igualmente necessários estudos adicionais para identificar as causas da não utilização de medicação no doente idoso (não prescrição, não adesão ou não aquisição), que podem dificultar as decisões terapêuticas no sentido da sua correcção.
A possibilidade de ocorrência de um viés de informação na identificação de omissões de prescrição, devido ao desconhecimento/esquecimento por parte dos doentes da sua medicação, foi minimizada através da confirmação da medicação de ambulatório nos diários clínicos e registos de enfermagem efectuados durante o internamento, registos esses onde habitualmente são escritas as informações do doente obtidas junto dos seus familiares. Ainda assim, uma vez que a medicação prescrita não foi confirmada junto da fonte de prescrição, por diversas razões (tais como alguns doentes consultarem diferentes médicos, não terem consultas médicas regulares apesar das suas co-morbilidades, ou casos de auto-medicação), reconhece-se que poderá existir algum viés de informação nos dados recolhidos.

Apesar destas limitações, os critérios START revelaram-se um instrumento útil de orientação da prescrição farmacológica em idosos, de acordo com a evidência das normas de orientação clínica actuais, sobretudo em termos de prevenção cardiovascular. De realçar, no entanto, que a individualização terapêutica, com identificação das causas das omissões de prescrição sinalizadas e intervenção para a sua possível correcção, requer julgamento clínico. Nem todas as intervenções farmacológicas beneficiam todas as pessoas da mesma forma, e muitas delas podem mesmo originar prejuízo e morbilidade. ${ }^{43}$ Num contexto de prevenção quaternária, que visa evitar o excesso de intervencionismo médico através da detecção de indivíduos em risco de sobretratamento, a decisão médica de prescrição deve fundamentar-se em princípios da proporcionalidade e da precaução iatrogénica (os ganhos esperados devem superar os eventuais danos). ${ }^{43}$ Estas decisões são particularmente complexas na população idosa, com um limite muito ténue entre a subutilização de medicamentos considerados necessários e o excesso de medicação.

Assim, os resultados deste estudo devem ser interpretados com precaução, tendo em conta que a informação da medicação prescrita não foi recolhida na fonte de prescrição, o que impossibilitou a identificação das causas das omissões detectadas.

As omissões detectadas neste estudo pelos critérios START não podem, de modo algum, ser consideradas erro médico. Os critérios START não devem ser utilizados para avaliar a prescrição médica pois não substi- 
tuem o julgamento clínico, mas antes orientar e fortalecer a tomada de decisões para optimização da terapêutica.

Numa era em que a utilização de medicamentos constitui a forma dominante de prestação de cuidados de saúde, sobretudo na população idosa, e em que a utilização simultânea de múltiplos medicamentos é muitas vezes necessária com base nas orientações terapêuticas estabelecidas, o estudo chama atenção para o problema de subutilização medicamentosa, mesmo em doentes polimedicados, com implicações preocupantes em termos de resultados em saúde. É de salientar que as omissões detectadas pelos critérios START ocorrem na sequência de eventos cardiovasculares agudos, que poderiam eventualmente ter sido prevenidos com a identificação mais precoce das mesmas. A literatura demonstra que uma abordagem terapêutica intensiva e multifactorial para modificação do risco cardiovascular nos idosos diminui a incidência de eventos cardiovasculares a longo prazo, sem interferir negativamente com a sua qualidade de vida. ${ }^{22,44}$

Assim, a incorporação dos critérios START em meio clínico, especialmente ao nível dos cuidados de saúde primários, pode constituir um importante instrumento para auxiliar a optimização da terapêutica na população idosa, com impacto na prevenção de admissões hospitalares por doença aguda.

\section{REFERÊNCIAS BIBLIOGRÁFICAS}

1. Hashimoto K, Tabata K. Population aging, health care and growth.J Popul Econ 2010 Mar; 23 (2): 571-93.

2. WHO, Preventing Chronic Diseases. A vital investment. 2005 [on-line] Disponível em: http://www.who.int/chp [acedido em 03/01/2011].

3. Carrilho MJ, Patrício L. A situação demográfica recente em Portugal. Rev Est Demográficos 2009; 46: 59-108. Disponível em: http://www.ine.pt/xportal/xmain? $x p i d=I N E \& x p g i d=i n e \_p u b l i c a-$ coes\&PUBLICACOESpub_boui $=81528281 \&$ PUBLICACOESte$\mathrm{ma}=55466 \&$ PUBLICACOESmodo $=2$ [acedido em 03/01/2011].

4. Cartwright A, Smith C. Elderly People, their medicines and their doctors. London, New York: Routledge; 1988.

5. Hajjar ER, Cafiero AC, Hanlon JT. Polypharmacy in elderly patients. Am J Geriatr Pharmacother 2007 Dec; 5 (4): 345-51.

6. Hughes CM. Medication non-adherence in the elderly: how big is the problem? Drugs Aging 2004; 21 (12): 793-811.

7. Hanlon JT, Lindblad Cl, Hajjar ER, McCarthy TC. Update on drug-related problems in the elderly. Am J Geriatr Pharmacother 2003 Sep; 1 (1): 38-43.

8. Unidade de Missão para os Cuidados Continuados Integrados. Problemas Relacionados com Medicamentos (PRM) no Idoso. Fev 2008. Dis- ponível em: http://www.socialgest.pt/_dlds/CCProblemasRelacionadosMedicamentosldoso.pdf [acedido em 03/01/2011].

9. Santos H, Iglésias P, Fernández-Llimós F, Faus MJ, Rodrigues LM. Segundo consenso de Granada sobre problemas relacionados com medicamentos: tradução intercultural de espanhol para português (europeu). Acta Med Port 2004 Jan-Fev; 17 (1): 59-66.

10. Wright RM, Sloane R, Pieper CF, Ruby-Scelsi C, Twersky J, Schmader KE, et al. Underuse of indicated medications among physically frail older US veterans at the time of hospital discharge: results of a cross-sectional analysis of data from the Geriatric Evaluation and Management Drug Study. Am J Geriatr Pharmacother 2009 Oct; 7 (5): 271-80.

11. Lindley CM, Tully MP, Paramsothy V, Tallis RC. Inappropriate medication is a major cause of adverse drug reactions in the elderly patients. Age Ageing 1992 Jul; 21 (4): 294-300.

12. Bates DW, Cullen DJ, Laird N, Petersen LA, Small SD, Servi D, et al. Incidence of adverse drug events and potential adverse drug events. Implications for prevention. JAMA 1995 Jul 5; 274 (1): 29-34.

13. Barry PJ, Gallagher P, Ryan C. Inappropriate prescribing in geriatric patients. Curr Psychiatry Rep 2008 Feb; 10 (1): 37-43.

14. Spinewine A, Schmader KE, Barber N, Hughes C, Lapane KL, Swine C, et al. Appropriate prescribing in elderly people: how well can it be measured and optimized? Lancet 2007 Jul 14; 370 (9582): 173-84.

15. Beers MH, Ouslander JG, Rollingher I, Reuben DB, Brooks J, Beck JC. Explicit criteria for determining inappropriate medication use in nursing homes. Arch Inter Med 1991 Sep; 151 (9): 1825-32.

16. Beers MH. Explicit criteria for determining potentially inappropriate medication use in elderly - an update. Arch Intern Med 1997 Jul 28; 157 (14): 1531-36.

17. Naugler $C T$, Brymer $C$, Stolee $P$, Arcese ZA. Development and validation of an improving prescribing in the elderly tool. Can J Clin Pharmacol 2000 Summer; 7 (2): 103-7.

18. Hanlon JT, Schmader KE, Samsa GP, Weinberger M, Uttech KM, Lewis IK, et al. A method assessing drug therapy appropriateness. J Clin Epidemiol 1992 Oct; 45 (10): 1045-51.

19. Wenger NS, Shekelle PG. Assessing care of vulnerable elders: ACOVE project overview. Ann Intern Med 2001 Oct 16; 135 (8 Pt 2) :642-6.

20. Gallagher P, Ryan C, Byrne S, Kennedy J, O'Mahony D. STOPP (Screening Tool of Older Person's Prescriptions) and START (Screening Tool to Alert Doctors to Right Treatment) - Consensus validation. Int J Clin Pharmacol Ther 2008 Feb; 46 (2): 72-83.

21. Barry PJ, Gallagher P, Ryan C, O'Mahony D. START (Screening Tool to Alert Doctors to the Right Treatment) - an evidence-based screening tool to detect prescribing omissions in elderly patients. Age Ageing 2007 Nov; 36 (6): 632-8.

22. Fourth Joint Task Force of the European Society of Cardiology and other societies on cardiovascular disease prevention in clinical practice. European guidelines on cardiovascular disease prevention in clinical practice: executive summary. Eur Heart J 2007 Oct; 28 (19): 2375-414.

23. Szadkowska I, Stanczyk A, Aronow WS, Kowalski J, Pawlicki L, Ahmed A, et al. Statin therapy in the elderly: a review. Arch Gerontol Geriatr 2010 Jan-Feb; 50 (1): 114-8.

24. Castilla-Guerra L, Fernández-Moreno MC, Alvarez-Suero J. Secondary stroke prevention in the elderly: new evidence in hypertension and hyperlipidemia. Eur J Intern Med 2009 Oct; 20 (6): 586-90. 
25. Kuijpers MA, van Marum RJ, Egberts AC, Jansen PA; OLDY (OLd people Drugs \& $\mathrm{dY}$ sregulations) Study Group. Relationship between polypharmacy and underprescribing. Br J Clin Pharmacol 2008 Jan; 65 (1): 130-3.

26. Steinman MA, Landefeld CS, Rosenthal GE, Berthenthal D, Sen S, Kaboli PJ. Polypharmacy and prescribing quality in older people. J Am Geriatr Soc 2006 Oct; 54 (10): 1516-23.

27. Broeiro P, Maio I, Ramos V. Polifarmacoterapia: estratégias de racionalização. Rev Port Clin Geral 2008 Set-Out; 24 (5): 625-31.

28. Amarenco P, Labreuche J, Lavallé P, Touboul PJ. Statins in stroke prevention and carotid atherosclerosis: systematic review and up-to-date meta-analysis. Stroke 2004 Dec; 35 (12): 2902-9.

29. European Stroke Organization (ESO) Executive Committee, ESO Writing Committee. Guideline for management of ischaemic stroke and transient ischaemic attack 2008. Cerebrovasc Dis 2008; 25 (5): 457507.

30. Smith SC Jr, Allen J, Blair SN, Bonow RO, Brass LM, Fonarow GC, et al. AHA/ACC guidelines for secondary prevention for patients with coronary and other atherosclerotic vascular disease: 2006 Update - endorsed by the National Heart, Lung, and Blood Institute. Circulation 2006 May 16; 113 (19): 2363-72.

31. Williams MA, Fleg JL, Ades PA, Chaitman BR, Miller NH, Mohiuddin SM, et al. Secondary prevention of coronary heart disease in the elderly (with emphasis on patients $>75$ years of age): an American Heart Association scientific statement from the council on clinical cardiology subcommittee on exercise, cardiac rehabilitation and prevention. Circulation 2002 Apr 9; 105 (14): 1735-43.

32. Erdmann E. Safety and tolerability of beta-blockers: prejudices and reality. Eur Heart J Suppl 2009 Mar; 11 (Suppl A): A21-A25.

33. Gibbons RJ, Abrams J, Chatterjee K, Daley J, Deedwania PC, Douglas JS, et al.ACC/AHA 2002 guideline update for the management of patients with chronic stable angina: summary article - a report of the American College of Cardiology/American Heart Association Task Force on practice guidelines (committee on the management of patients with chronic stable angina). Circulation 2003 Jan; 107 (1): 149-58.

34. Fox K, Garcia MA, Ardissino D, Buszman P, Camici PG, Crea F, et al. Guidelines on the management of stable angina pectoris: executive summary: The task force on the management of stable angina pectoris of the European Society of Cardiology. Eur Heart J 2006 Jun; 27 (11): 1341-81.

35. US Department of Health and Human Services. National Institutes of Health, National Heart, Lung, Blood Institute - The seventh report of the Joint National Committee on prevention, detection, evaluation, and treatment of high blood pressure (JNC7). 2004. Disponível em: http://www.nhlbi.nih.gov/guidelines/hypertension/ [acedido em 03/01/2011].

36. Mancia G, De Backer G, Dominiczak A, Cifkova R, Fagard R, Germano $\mathrm{G}$, et al. 2007 Guidelines for the management of arterial hypertension: the Task Force for the Management of Arterial Hypertension of the European Society of Hypertension (ESH) and the European Society of Cardiology (ESC). Eur Heart J 2007 Jun ; 28 (12): 1462-536.

37. Gage BF, Boechler M, Doggette AL, Fortune G, Flaker GC, Rich MW, et al.Adverse outcomes and predictors of underuse of antithrombotic therapy in Medicare beneficiaries with chronic atrial fibrillation. Stroke 2000 Apr; 31 (4): 822-7.
38. Fuster V, Rydén LE, Cannom DS, Crijns HJ, Curtis AB, Ellenbogen KA, et al. ACC/AHA/ESC 2006 guidelines for the management of patients with atrial fibrillation: full text - A report of the American College of Cardiology / American Heart Association Task Force on practice guidelines and the European Society of Cardiology Committee for Practice Guidelines (Writing Committee to revise the 2001 guidelines for the management of patients with atrial fibrillation) developed in collaboration with the European Heart Rhythm Association and the Heart Rhythm Society. Europace 2006 Sep; 8 (9): 651-745.

39. Sirois C, Moisan J, Poirier P Grégoire JP. Underuse of cardioprotective treatment by the elderly with type 2 diabetes. Diabetes Metab 2008 Apr; 34 (2): 169-76.

40. Sigal R, Malcolm J, Meggison H. Prevention of cardiovascular events in diabetes. Clin Evid 2005; 14: 501-21.

41. Rydén L, Standl E, Bartnik M, Van den Berghe G, Betteridge J, de Boer MJ, et al. Guidelines on diabetes, pre-diabetes, and cardiovascular diseases: executive summary. The Task Force on Diabetes and Cardiovascular Diseases of the European Society of Cardiology (ESC) and of the European Association for the Study of Diabetes (EASD). Eur Heart J 2007 Jan; 28 (1): 88-136.

42. Antithrombotic Trialists' (ATT) Collaboration. Aspirin in the primary and secondary prevention of vascular disease: collaborative meta-analysis of individual participant data from randomised trials. Lancet 2009 May; 373 (9678): 1849-60.

43. Melo M. A prevenção quaternária contra os excessos da Medicina. Rev Port Clin Geral 2007 Mai-Jun; 23 (3): 289-93.

44. Janssen PG, Gorter KJ, Stolk RP, Rutten GE. Randomised controlled trial of intensive multifactorial treatment for cardiovascular risk in patients with screen-detected type 2 diabetes: 1-year data from the ADDITION Netherlands study. Br J Gen Pract 2009 Jan; 59 (558): 43-8.

\section{CONFLITOS DE INTERESSE}

As autoras declaram não existirem conflitos de interesse na elaboração deste artigo.

\section{ENDEREÇO PARA CORRESPONDÊNCIA}

Ana Filipa Macedo

Faculdade de Ciências da Saúde

Universidade da Beira Interior

Av. Infante D. Henrique, 6200-506 Covilhã

Email: filipa@fcsaude.ubi.pt

Recebido em 12/09/2010

Aceite para publicação em 15/02/2011 


\section{ABSTRACT}

\section{PRESCRIBING OMISSIONS OF CARDIOVASCULAR RISK MANAGEMENT THERAPY IN ACUTELY-ILL ELDERLY PATIENTS ADMITTED AT A STROKE UNIT}

Goals: To quantify and characterize prescribing omissions of cardiovascular risk management therapy using START (Screening Tool to Alert Doctors to the Right Treatment) criteria.

Type of Study: Descriptive observational study Local: Stroke Unity of the "Centro Hospitalar da Cova da Beira (CHCB)". Population: Elderly patients (age $\geq 65$ years) admitted with acute cardiovascular disease.

Methods: Medical records from elderly patients admitted between October and December 2009 were consulted and the START criteria applied to data on prescribed medicines.

Results: Our study included 56 elderly in-patients. We detected 63 prescribing omissions in $69,8 \%$ of these elderly (average of 1,19 omissions by patient), from which $74,5 \%(n=38)$ were corrected. Prescribing omissions were also detected in $80,9 \%$ of patients taking 5 or more medications simultaneously. In 10 patients, 13 omissions detected at hospital admission were not corrected and in 3 patients, 3 new prescribing omissions were detected.

Conclusions: The prevalence of omission of evidence-based appropriate drug therapy in cardiac acutely-ill hospitalized elderly patients is high. START criteria are a useful instrument to validate essential drug therapy, particularly in relation to cardiovascular disease prevention.

Keywords: Elderly; Adverse Drug Reaction; Drug Prescriptions; Stroke; Cardiovascular Disease. 